\title{
Bioanalysis
}

\section{Implementation of a novel ultra fast metabolic stability analysis method using exact mass TOF-MS}

\begin{abstract}
Aim: Increasing numbers of compounds requiring stability data means highly optimized methods capable of rapid turnaround are desirable during early discovery. Materials and methods/results: An advanced, generic analytical workflow for metabolic stability has been developed that utilizing ballistic gradient LC (sub 1 min run times), exact mass TOF-MS (Waters Xevo-G2-XS Q-TOF) and automated data processing (Waters UNIFI software) allowed for rapid integration and interpretation of all data produced, eliminating the need for method development and manual processing. We can analyze and process 96 compounds across two species in quadruplicate in a 24-h period with no method development. Conclusion: An advanced bioanalytical workflow has increased our capacity threefold and reduced our instrument/processing needs threefold.
\end{abstract}

First draft submitted: 12 July 2016; Accepted for publication: 5 December 2016; Published online: 11 January 2017

Keywords: automated data $\bullet$ automation $\bullet$ ballistic gradient $\bullet$ discovery processing

- efficiency $\bullet$ high resolution $\bullet$ mass spectrometry $\bullet$ metabolic stability

Drug metabolism and pharmacokinetics (DMPK) in drug discovery has always played a central role in optimizing 'drug-like' properties for new chemical entities (NCEs) [1,2]. In order to provide useful and valuable data in a timely manner, DMPK assays have had to conform to mid-to-high throughput formats, whenever possible, to keep pace with the speed of high-throughput medicinal chemistry, which allows medicinal chemists to synthesize large numbers of NCEs in a short amount of time. This increased volume is a challenge to discovery DMPK groups with regard to speed of data turnaround time $[3,4]$.

In vitro DMPK screens have been developed and have shown to be very effective in guiding candidate optimization and preventing selection of compounds with poor 'druglike' properties, including metabolic stability assays, compound solubility assays and permeability assays. The major advantages of using these in vitro screens include the ability to test large numbers of compounds (NCEs) early in drug discovery; many of these assays are adaptable to high throughput screening and automation platforms; and screening assays can utilize human derived cells or cellular systems which increase the relevance and predictability in humans $[5,6]$.

One of the most commonly performed in vitro DMPK assays during early discovery is metabolic stability [7]. This assay is used to predict the susceptibility of compounds to metabolism primarily by the liver, and helps eliminate compounds with metabolic liabilities early in the process. Because of the importance of this assay and the increasing number of compounds requiring metabolic stability data [8], highly optimized methods capable of rapid turnaround are desirable during early stage drug discovery.

Commonly, data for metabolic stability assays are generated using HPLC cou-
Joseph D Manna', Samantha J Richardson ${ }^{1}$ \& Mehran F Moghaddam $*, 1$

'Department of Drug

Metabolism \& Pharmacokinetics, Celgene Corporation, 10300 Campus Point Dr., San Diego, CA 92121, USA

*Author for correspondence:

Tel.: +1 8587954791

Fax: +1 8585528775

memo@oroxbios.com 
(A)

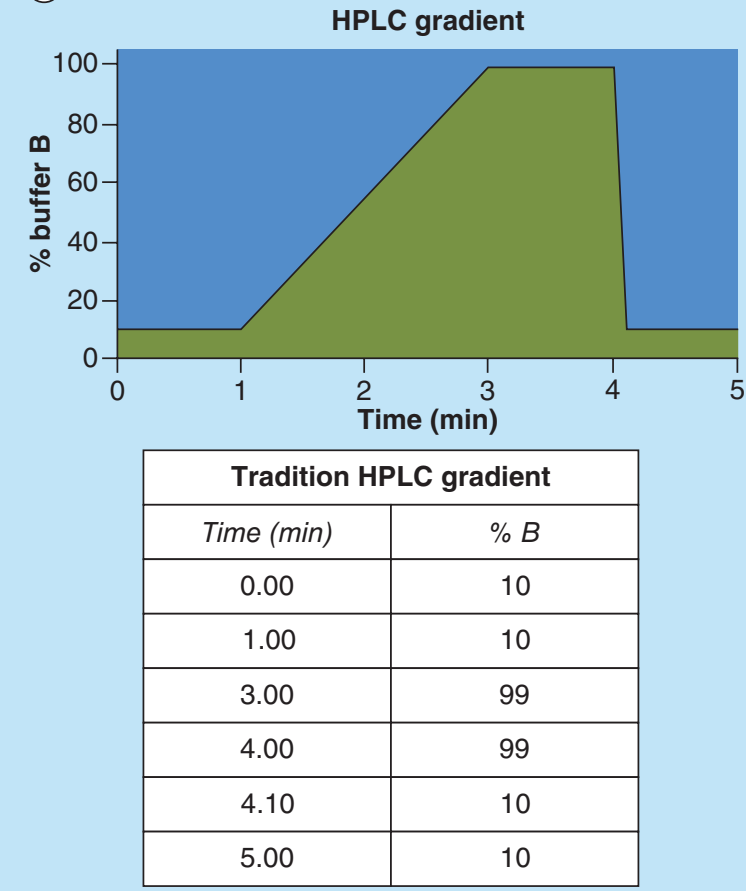

(B)

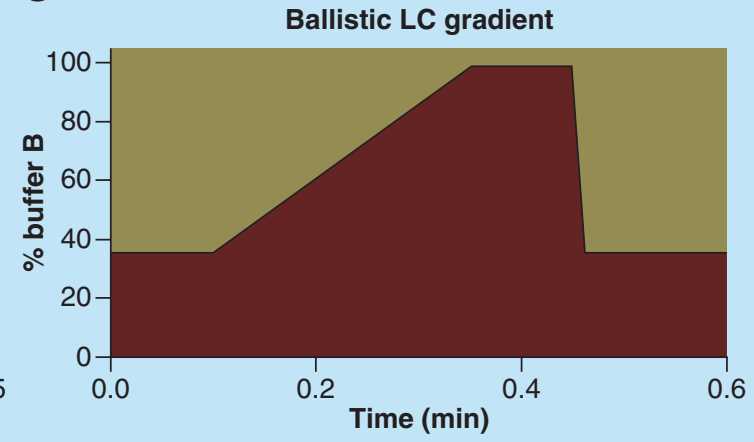

\begin{tabular}{|c|c|}
\hline \multicolumn{2}{|c|}{ UFast-MS gradient } \\
\hline Time $($ min) & $\%$ B \\
\hline 0.00 & 25 \\
\hline 0.10 & 25 \\
\hline 0.35 & 99 \\
\hline 0.45 & 99 \\
\hline 0.46 & 25 \\
\hline 0.60 & 25 \\
\hline
\end{tabular}

Figure 1. Comparison of traditional HPLC gradient traces with UFast-MS gradient. (A) Represents the traditional HPLC gradient run for typical metabolic stability assays. (B) Represents the sub 1-min ballistic LC gradient developed for UFast-MS.

pled with a triple quadrupole mass spectrometer (LC-MS/MS). One major bottleneck of this workflow is the need to develop methods for each compound independently before analysis, which requires both labor and instrument time in order to achieve. Many modern DMPK groups have taken advantages of improvements in LC technology to help improve analytical throughput. Ultra-performance liquid chromatography (UPLC or ballistic-LC) has allowed for improvements in resolution, sensitivity and speed compared with traditional HPLC. These significant increases in resolution and sensitivity have allowed for noticeable reductions in analytical run time allowing UPLC to become a powerful analytical tool for highthroughput analysis. For instance, Apollonio et al. [9] demonstrated the power of UPLC when they evaluated a number of different amphetamine-like compounds in a whole blood extract. Utilizing this technology, they were able to separate nine different compounds in less than 3 min [9]. Additionally, other groups have shown the ability to reduce pharmacokinetic (PK) analytical run times using UPLC from 5 to 2 min per sample, demonstrating the power of UPLC and the ability to decrease run time without sacrificing data quality [1013]. Additionally, because of the time needed for compound optimization and the number of samples generated during high-throughput screens, many groups couple UPLC-MS/MS analysis with sample pooling strategies [14-18]. For example, $\mathrm{Xu}$ et al. developed a pooling strategy based on the cLogP values of compounds to ensure sufficient chromatographic separation using UHPLC-MS/MS after individual incubation in 96-well plate format. Though they were able to decrease the analytical run time by $50 \%$, they still had a compound fail rate of $20 \%$, which they alleviated by developing custom data processing software [18]. These pooling strategies have been employed to help decrease analytical run time, however the approach does introduce a number of potential problems; including variability in analysis for poorly ionizable samples due to dilution during pooling, compound to compound interference (e.g., ion suppression) and potential coelusion of isomers [14-18].

Furthermore, the emergence of high-resolution mass spectrometers (HRMS), such as Orbitrap or quadrupole TOF systems, has led to the development of new analytical approaches for different types of metabolism studies. The majority of newly developed analytical methods focus on the identification of unknown metabolites without having to use multiple instruments and modes of analysis, for example, neutral loss or MS3 scans, for identification and elucidation [19-21]. With the improvements in both MS technology as well as the LC techniques mentioned above, groups have 
begun to multiplex analyses in order to determine as much metabolic data as possible. O'Connor et al. took the approach of combining ballistic gradient LC techniques with HRMS to determine both metabolic stability and metabolic identification by performing full scan data acquisition over a 3 min gradient, allowing for extracted ion chromatograms to be generated for quantitative metabolic stability as well as unknown metabolite information [1]. Though this new method does produce significant amounts of data, it still does not achieve the throughput necessary for modern drug discovery. Expanding on O'Connor et al., Nagele and Fandino utilized column-switching techniques in connection with TOF-MS analysis to determine both the metabolic stability of compounds as well as the metabolic profile of these unknown compounds to determine both the metabolic stability of NCEs as well as the metabolic profile for these compounds [22]. Similar to previous methods, the amount of time necessary for the determination of both metabolic stability data and identification of unknown metabolites is not suitable for the modern drug discovery workflows.

Our group has taken a different approach in our use of HRMS and ballistic LC technology to develop a tailored analytical approach that removes many of the variables/problems with traditional LC-MS/MS analysis and has the speed necessary to determine metabolic stability data in a timely manner. By taking advantage of the improvements in both chromatography and mass spectrometer technology, we have developed a significantly improved analytical workflow which couples ballistic gradient LC and exact mass TOF-MS. This eliminates the need for mass spectrometric method development and streamlines the metabolic stability workflow, helping in providing crucial data to discovery project teams at a rapid pace to guide structure-activity relationships of large sets of compounds in a timely manner. It is obvious that use of ballistic gradient LC would speed up the run time and that replacing the tandem with TOF-MS would eliminate development of MS/MS methodology. In fact, other groups have coupled these two concepts for various reasons $[1,12,13]$. However, our manner of combining various components and the custom designed IT solution that needed to be developed due to lack of their commercial availability did not exist in the literature, and therefore, constituted a novel approach.

\section{Materials \& methods}

\section{Chemicals}

HPLC grade water, acetonitrile (ACN), methanol $(\mathrm{MeOH})$, DMSO, formic acid, $\beta$-nicotinamide adenine dinulceotide reduced tetrasodium salt hydrate (NADPH), L-glutathione reduced form (GSH), uridine 5'-diphosphoglururonic acid trisodium salt (UDPGA), adenosine 3'-phosphate 5'-phosphosulfate lithium salt hydrate (PAPS), Trizma ${ }^{\circledR}$ pre-set crystals (Tris), magnesium chloride hexahydrate $\left(\mathrm{MgCl}_{2}\right)$, sodium hydroxide $(\mathrm{NaOH})$ and 7-ethoxycoumacrin (7-EC) were all obtained from Sigma Chemical Company (St. Loius, MO, USA). Rat and human S9 liver fractions were purchased from BD Biosciences (CA, USA). All proprietary drug discovery compounds that are tested in our routine metabolic stability assay are synthesized by the Medicinal Chemistry Department at Celgene Corporation. These compounds are all small molecules with a diverse array of chemical matter and molecular weights ranging from 200 to 900 .

\section{High-throughput liver $\$ 9$ metabolic stability assay}

A 384-well high-throughput method was developed on a TECAN Evo liquid handler (NC, USA) equipped with a liquid handling arm with 96-well disposable tip handling, a robotic arm for plate movement, a temperature controlled deck (maintained at $4^{\circ} \mathrm{C}$ ), temperature
(A)

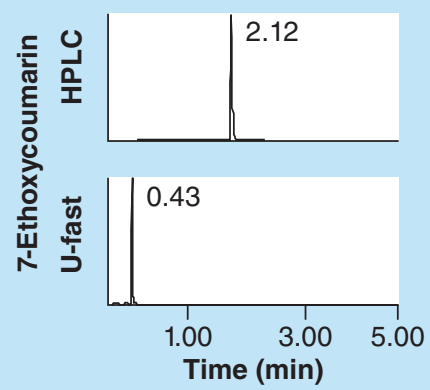

(B)

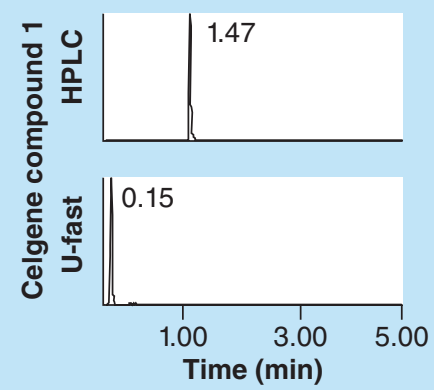

(C)

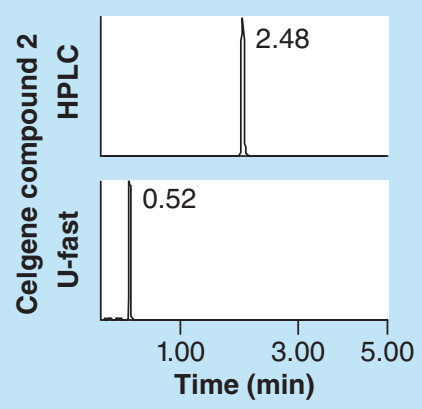

Figure 2. Comparison of HPLC-MS traces with UFast-MS method traces. Comparison of (A) 7-EC and two Celgene compounds 1 and 2 (B \& C, respectively) show comparable quality of separation with superior speed for UFast-MS compared to HPLC. 


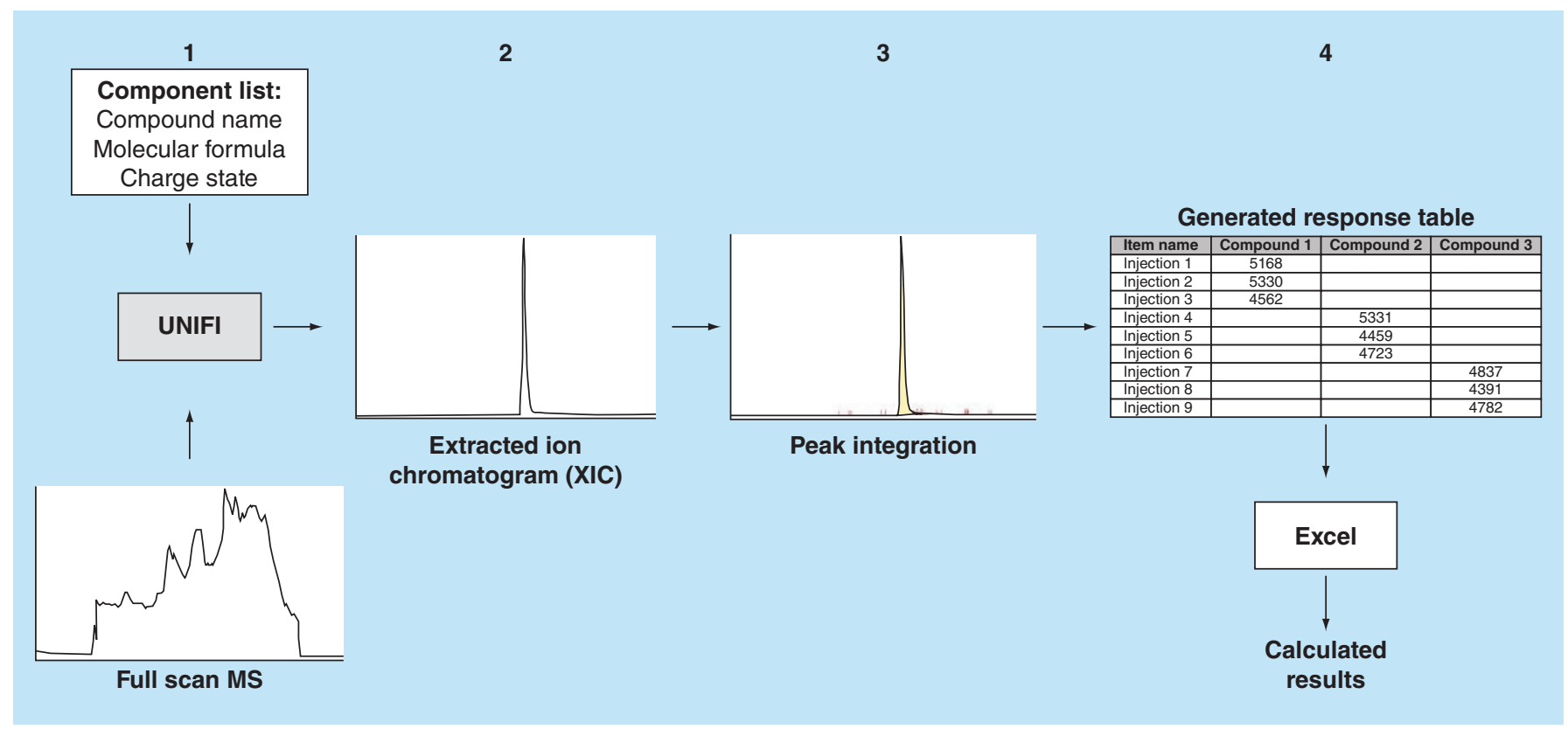

Figure 3. UNIFI workflow for data acquisition and processing. Data acquisition and processing was performed on an analysis of 7-EC (A). Full scan data are acquired and processed against a known component list. (B) Extracted ion chromatogram is generated for all identified components (C) peaks are integrated for response and (D) all processed responses are exported into Excel for further calculations.

controlled orbital shakers and a high speed centrifuge. This high-throughput method allows for the analysis of 96 compounds in quadruplicate across two species. The robotic method was validated using a commercially available compound, 7-EC, with known metabolic profiles (both Phase I and II) in both human and rat $S 9$ fractions. The $S 9$ fractions were chosen for metabolic stability over microsomes or hepatocytes because of the reasons presented in Richardson et al. [20]. In summary, utilizing $S 9$ fractions allows for a more accurate representation of Phase I and II metabolites generated in vivo compared with microsomes and without the expense of running hepatocytes.

The S9 protein and cofactor concentrations were optimized to match the results of hepatocyte stability, as previously described [23]. Final rat and human liver S9 protein concentrations were 1.1 and $1.2 \mathrm{mg} / \mathrm{ml}$, respectively. These protein concentrations were chosen to ensure our control compound (7-EC) had percentage remaining values of $20-35 \%$ for human and $50-65 \%$ for rat liver S9 after a 60 min incubation. A mixture of four cofactors was used in order to stimulate both Phase I (NADPH) and Phase II (UDPGA, PAPS and GSH) metabolism. The final concentrations of NADPH, UDPGA and GSH were $1,0.5$ and $2.5 \mathrm{mM}$, respectively, while that of PAPS was $0.05 \mathrm{mg} / \mathrm{ml}$.

A $200 \mathrm{mM}$ tris buffer ( $\mathrm{pH}$ 7.4) containing $2 \mathrm{mM}$ $\mathrm{MgCl}_{2}$ was prepared in HPLC grade water and adjusted with $1 \mathrm{M} \mathrm{NaOH}$. Stock solutions of control (7-EC) and test compounds were prepared at $300 \mu \mathrm{M}$ in DMSO:ACN (1:1, v:v). NADPH, UDPGA and GSH solutions were prepared at 40,20 and $2 \mathrm{mM}$, respectively while PAPS was prepared at $2 \mathrm{mg} / \mathrm{ml}$, all in tris buffer prior to mixing together in a 1:1:1:1 (v:v:v:v) ratio before use. A total of $100 \mu \mathrm{l} /$ well of S9s were preincubated for $5 \mathrm{~min}$ at $37^{\circ} \mathrm{C}$ in tris buffer prior to addition of $1 \mu \mathrm{l}$ of control/test compounds. Reactions were initiated by addition of cofactor mixture. At two time points, $0 \mathrm{~min}$ and $60 \mathrm{~min}, 25 \mu \mathrm{l}$ aliquots of the sample mixture was removed and quenched by addition of $50 \mu \mathrm{l}$ of $\mathrm{ACN}: \mathrm{MeOH}(1: 1, \mathrm{v}: \mathrm{v})$. The plate of quenched samples was centrifuged at $10,000 \times g$ for $10 \mathrm{~min}$ to pellet the precipitated protein. Supernatant was diluted $(1: 1, \mathrm{v}: \mathrm{v})$ with LC-MS grade water prior to injection onto LC-QTOF-MS. Percentage remaining and SD were calculated for all samples.

\section{Bioanalysis/ultra-fast LC/MS conditions}

A newly developed analytical workflow utilizing ballistic gradient technique coupled with an exact mass QTOFMS was employed for analysis of all samples. Samples ( $3 \mu \mathrm{l}$, flow-through needle injections) were analyzed using a ballistic gradient. Mobile phases used for chromatographic separation were as follows: mobile phase $\mathrm{A}$ (water-formic acid [100:0.001, v/v]) and mobile phase B (ACN-formic acid [100:0.001, v/v]). All samples were separated using an Imtakt Unison C18 LC column (20 $\mathrm{mm} \times 3 \mathrm{~mm}, 3 \mu \mathrm{m}$ particle size), positioned between the autosampler and switching valve, which allowed flow to be directed between waste and mass analyzer. Samples 
were injected onto the column with an initial condition of $75 \%$ mobile phase $A$ at a flow rate of $1 \mathrm{ml} / \mathrm{min}$. Sample was diverted to waste for $0.05 \mathrm{~min}$ before being switched and a linear gradient from $0.1 \mathrm{~min}$ to $0.35 \mathrm{~min}$ increasing organic from $25 \%$ mobile phase B to $99 \%$ mobile phase $\mathrm{B}$, which was then kept constant for $0.1 \mathrm{~min}$ to allow sample elution. The valve was switched to waste at $0.5 \mathrm{~min}$ and pumps returned to initial conditions. The entire LC/MS run time was $0.6 \mathrm{~min}$.

All compounds were detected using exact mass LC/MS. Compounds were analyzed on a Waters Xevo-G2-XS Q-TOF-MS with a Waters I-class LC system. All instruments were controlled using Waters software package, UNIFI ${ }^{\circledR}$ Scientific Information System (Waters Corp. Milford, MA, USA) . Exact mass analysis was performed in positive ion electrospray in sensitivity analysis mode. Source conditions were as follows: source temperature and desolvation temperature were 120 and $600^{\circ} \mathrm{C}$, respectively, cone gas flow and desolvation gas flow were 50 and 1000 l/h, respectively, cone voltage was $40 \mathrm{~V}$ and capillary voltage was $0.80 \mathrm{kV}$. TOF parameters were as follows: full scan analysis was performed with a mass range from 150 to $950 \mathrm{amu}$ with a scan rate of $0.15 \mathrm{~ms}$.

\section{Data processing}

During analysis, data were processed using a peak picking software developed by Waters called UNIFI.
Molecular formula, charge state and name of all test compounds were uploaded into the UNIFI software. The software then takes the molecular formula for each test component and calculates exact masses. Once data were generated, UNIFI utilizes a proprietary peak picking algorithm to identify peaks based on retention time, $\mathrm{m} / \mathrm{z}$ and intensity (3D-peak picking). Once all $\mathrm{m} / \mathrm{z}$ components are identified, the samples are compared with the uploaded exact mass component list and UNIFI identifies all components in each sample and generates a table of all peak areas from the 3D-MS traces. This peak area table is used to calculate 'percent remaining', described as follows: peak area of test compound detected at $60 \mathrm{~min}$ was divided by the peak area detected at $0 \mathrm{~min}$, value was converted to percentage and reported as 'percent remaining'. UNIFI data processing algorithm is described in greater detail in Componentization Following 3D-Peak Detection in the UNIFI Scientific Information System [24].

\section{Results \& discussion}

\section{Bioanalysis/Ultra-Fast (UFast) LC/MS analysis}

In order to analyze all the samples generated in the high-throughput metabolic stability assay in a timely manner, we needed to develop a new LC-MS method that allowed the analysis of 1152 samples in $24 \mathrm{~h}$. Our original method employed standard HPLC methods, which produced clean and robust data but usually had
(A)

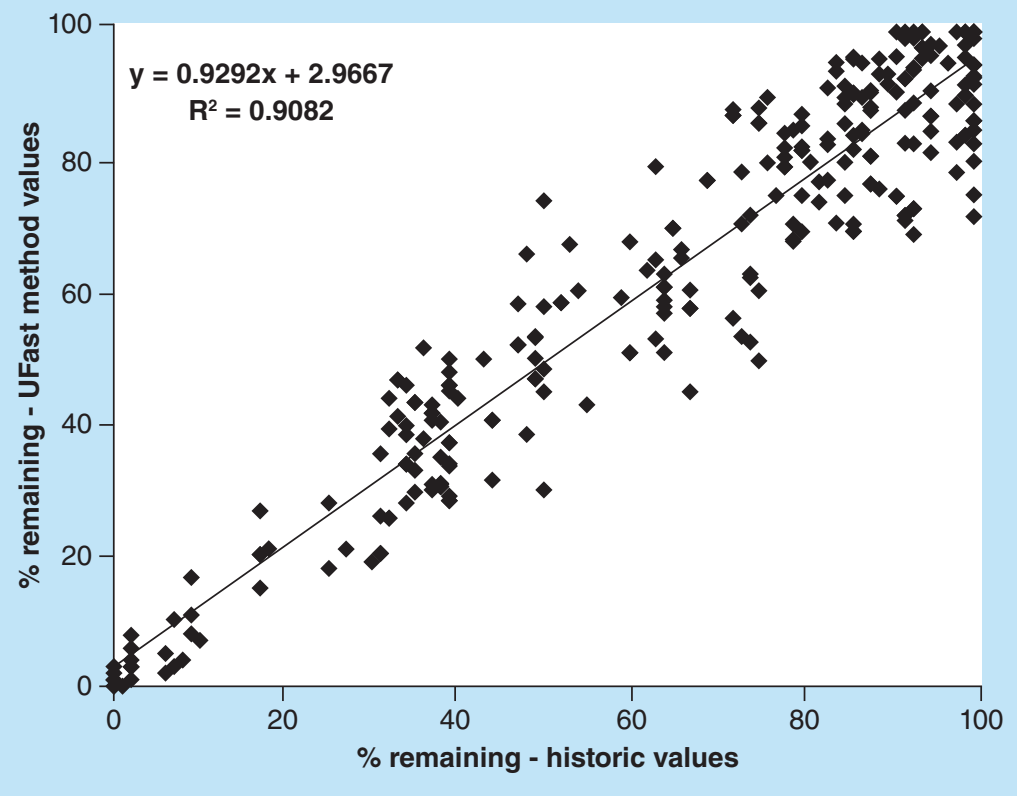

(B)
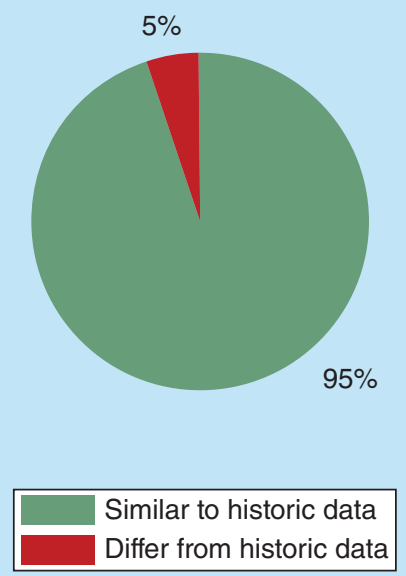

Figure 4. Comparison of historical stability data versus new stability workflow. (A) Correlation curve comparing the percent remaining determined from historical metabolic stability assays and newly generated values using UFast-MS based methods. Trend line for all data, $y=0.9292 x+2.9667$ with an $R^{2}=0.91$ and a $p$-value $<0.0001$. (B) Comparison of overall similarity between the two methods. 
Table 1. Comparison of metabolic stability data.

\begin{tabular}{|c|c|c|}
\hline Compound & $\begin{array}{l}\text { Historic stability data } \\
\% \text { Remaining } \pm \text { SD }(\% C V)(n=3)\end{array}$ & $\begin{array}{l}\text { UFast-MS stability data } \\
\% \text { Remaining } \pm \text { SD }(\% C V)(n=4)\end{array}$ \\
\hline 7-Ethoxycoumarin (7-EC) & $59 \pm 8(14)$ & $58 \pm 7(12)$ \\
\hline Celgene compound 1 & $93 \pm 1(1)$ & $95 \pm 3(3)$ \\
\hline Celgene compound 2 & $84 \pm 13(15)$ & $95 \pm 9(9)$ \\
\hline Celgene compound 3 & $86 \pm 3(3)$ & $90 \pm 7(7)$ \\
\hline Celgene compound 4 & $78 \pm 1(1)$ & $82 \pm 3(4)$ \\
\hline Celgene compound 5 & $91 \pm 2(2)$ & $96 \pm 2(2)$ \\
\hline Celgene compound 6 & $2 \pm 0(0)$ & $6 \pm 1(11)$ \\
\hline Celgene compound 7 & $95 \pm 9(9)$ & $85 \pm 2(3)$ \\
\hline Celgene compound 8 & $37 \pm 2(5)$ & $41 \pm 1(3)$ \\
\hline Celgene compound 9 & $49 \pm 2(4)$ & $50 \pm 3(6)$ \\
\hline Celgene compound 10 & $6 \pm 0(0)$ & $13 \pm 0(0)$ \\
\hline Celgene compound 11 & $43 \pm 0(0)$ & $37 \pm 1(2)$ \\
\hline Celgene compound 12 & $76 \pm 1(1)$ & $88 \pm 3(3)$ \\
\hline Celgene compound 13 & $48 \pm 1(2)$ & $56 \pm 4(8)$ \\
\hline Celgene compound 14 & $6 \pm 0(0)$ & $6 \pm 2(27)$ \\
\hline Celgene compound 15 & $65 \pm 0(0)$ & $65 \pm 5(8)$ \\
\hline Celgene compound 16 & $36 \pm 1(3)$ & $41 \pm 2(5)$ \\
\hline Celgene compound 17 & $60 \pm 10(17)$ & $72 \pm 8(11)$ \\
\hline Celgene compound 18 & $36 \pm 3(8)$ & $46 \pm 6(13)$ \\
\hline Celgene compound 19 & $91 \pm 1(1)$ & $100 \pm 2(2)$ \\
\hline Celgene compound 20 & $87 \pm 2(2)$ & $76 \pm 6(8)$ \\
\hline
\end{tabular}

a run time of about 3-5 min (Figure 1A). Utilizing these methods to analyze our 384-well high-throughput stability samples, it would have taken anywhere from 3 to 4 days to analyze all samples generated. To solve this problem, we utilized an ultra-fast LC-MS system to generate an LC gradient method that could be run in $36 \mathrm{~s}$ (Figure 1B). Figure 2 compares multiple LC-MS traces generated from our traditional HPLC method compared with our newly developed UFastMS method. The new UFast-MS method which produces traces that have an excellent peak shape and width (6s), show comparable chromatographic separation as traditional HPLC methods, and does not sacrifice on the quality of the data produced. Due to the fast nature of this method, one concern that may arise is the potential for compounds that are highly hydrophilic to be diverted in the short time sample is sent to waste. Comparing the physical chemical properties (cLogP) of approved drugs published and patented from a multitude of different companies, it is apparent that the optimal range for hydrophilicity exists in a range of cLogP between 2 and 5, with the majority demonstrat- ing more hydrophobic properties [25-27]. Taking this historical perspective into consideration, our group felt that any compounds that are too hydrophilic to be retained on the column would subsequently fail later in drug discovery due to other pharmacokinetic/dynamic issues.

Coupling this new LC method with high-resolution MS technology, we have eliminated the need for method development of every compound tested, which is required for traditional triple quad MS/MS analysis. This change from triple quad MS analysis to exact mass analysis will decrease the time and manpower necessary to develop methods for analysis. By taking advantage of the precision of our exact mass instrument in combination with the processing capabilities of the UNIFI software, we are able to use this generic full scan LC-MS for the analysis of all compounds tested in our metabolic stability assay. In this approach, each compound's molecular formula was entered, the software generated the corresponding exact mass and queued the TOF mass spectrometer data to look for that mass, extract the mass chromatogram and inte- 
grate and report the data automatically. Actual utility of this approach for studying large number of newly synthesized compounds has not been demonstrated under drug discovery setting before.

\section{Data processing}

The amount of data generated in a single UFast-MS method can vary between 1000 and 1200 samples over 90-96 different compounds. This amount of data processing can be difficult, time consuming and open to error. To rectify this potential difficulty, we took advantage of the processing capabilities of UNIFI software. This software allows us to operate our LC-MS system as well as simultaneously process samples during runs. As demonstrated in Figure 3, full scans generated on the LC-MS are processed against a set of compound molecular formulas input into the UNIFI software (e.g., compounds synthesized by medicinal chemistry are screened/input into this software, utilizing the molecular formula provided by the chemists). UNIFI then compares the exact mass of the input compounds and generates an extracted ion chromatogram for identified compounds. The peaks generated in the extracted ion chromatogram are then processed for response and all identified compounds are presented in table format ready for export into Excel ${ }^{\circledR}$. From here we have developed an internal Excel processing macro that allows us to parse all responses and compare to a known plate map of compounds. These parsed data then calculate all relevant parameters for metabolic stability; for example, percent remaining, SD and percent $\mathrm{CV}$.
High-throughput metabolic stability utilizing UFast-MS method techniques

Once we developed our generic analytical workflow, we began evaluating the method for screening purposes. We utilized our high-throughput metabolic stability screening assay as a way to evaluate the robustness of the UFast-MS method. Validation was conducted by comparing historic metabolic stability results generated from traditional triple quad MS techniques and comparing with the results generated from our new workflow. Figure 4 shows a comparison of percent remaining determined by historical stability techniques and our newly developed UFast-MS workflow. There is a good correlation (slope of the trend line is 0.9292$)$, low distribution of values $\left(R^{2}=0.91\right)$ and a high statistical significance $(\mathrm{p}<0.0001)$ between data generated from our historic stability assay and our newly developed UFast-MS method. Additionally, of the approximately 280 compounds evaluated, 95\% of them 'binned' similarly to values obtained from our historic data (Table 1 \& Supplementary Figure 1). Importantly, the quality of the data generated was not sacrificed in order to achieve increased throughput and speed. Table 1 shows that the UFast-MS method produced high-quality data with very low variability (SD and $\% \mathrm{CV}$ ).

Additionally, of the $5 \%$ that did not match, many were similar but not 'binned' according to our criteria. Internally, compound stability is 'binned' into three groups; metabolically stable ( $>70 \%)$, moderately stable $(70-30 \%)$ and unstable $(<30 \%)$ [28]. For example, in Table 1, Celgene compound 17 had stability numbers that were on the cusp of binned groups. For the historic
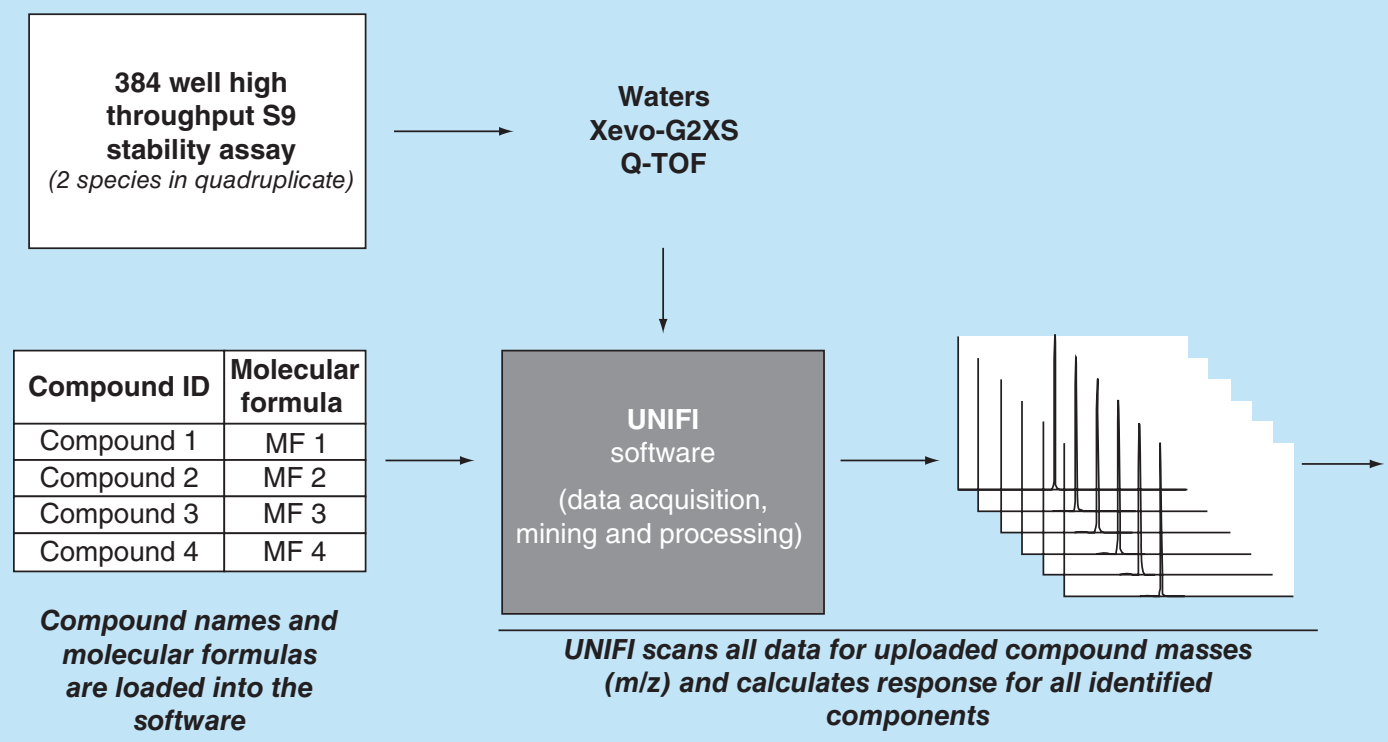

$\%$ remaining, standard deviation and $\% \mathrm{CV}$

Figure 5. Summary of UFast-MS metabolic stability workflow. 
method the number measured was $60 \%$ remaining while the UFast-MS method measured $72 \%$ remaining. These numbers are similar but are binned in different groups, making them not similar based on our criteria. Additionally, some compounds (2-5\%) were not detected by the MS due to issues with ionization. In instances where this happens, we resort back to our traditional triple quad tuning method to verify issues of ionization. In all cases, there is no detectable signal on any instrumental platform and hence no data generated.

\section{Conclusion}

We successfully developed a novel high-throughput bioanalytical workflow (Figure 5) to address throughput challenges for metabolic stability assays in the fast pace discovery setting. While the technologies used have been reported on previously, [1,14-18] other groups have not reported on the combination of these techniques as well as the implementation of custom-designed solutions (e.g., automated UNIFI processing, data parsing solution) to address throughput challenges quite in the same way. Our newly developed UFast-MS method removes the need for any method development allowing for immediate analysis of samples with no downtime for MS optimization. By utilizing ballistic gradient techniques, we were able to develop a sub 1 min LC gradient chromatographic method and coupled with high resolution TOF-MS for the analysis of metabolic stability samples. This method demonstrates numerous advantages over traditionally LC-MS/MS analysis. Commonly, data for metabolic stability assays are generated using LC-MS/MS analysis. Though these methods are robust and reproducible, they need time to develop methods for each compound in the analysis, which requires human and/or instrument time in order to achieve [14-18].

Additionally, because of the time needed for method development, many groups utilize a sample pooling strategy in order to reduce total run time. Though pooling strategies do create fewer samples for analysis, they introduce potential problems allowing for compound to compound interference [14-18]. By utilizing improvements in LC technologies, we were able to develop a sub 1 min method which removes the need for sample pooling without any impact on total sample run time. By ensuring that each compound of interest is run independently of others guarantees there will be no compound to compound interference, removing any concerns from pooling strategies.

Lastly, data generated from these LC-MS/MS methods usually require user input in order to ensure processing methods for each compound analyzed are correct. UFast-MS solves this problem by taking advantage of the exact mass capability of the TOF and the processing power of UNIFI software. This allows for processing of samples simultaneously with data acquisition, reducing the need for processing method validation and allowing for faster turnaround of calculated data.

This newly developed generic method has the ability to analyze the stability of 96 compounds in quadruplicate across two species in less than $24 \mathrm{~h}$, removing the need for method optimization that is required in traditional MS/MS methods and minimizing data processing. This method demonstrates significant advantages over traditional triple quadrupole based methods, which include: ballistic LC allows for significant reductions in analytical run time to less than a minute/injection; eliminates the need for sample pooling; removes the risk of ion suppression; high resolution TOF-MS analysis removes the need for MS/MS method development and optimization for all compounds; and automated data processing allows for rapid integration and interpretation of all data generated. This new method has increased our capacity threefold and reduced our instrument/processing needs by threefold with no impact on the quality of data produced.

\section{Future perspective}

In early drug discovery, high-throughput assays have become common place in order to screen the large amount of compounds against new targets. With the importance of assessing the metabolic liabilities of compounds early in the discovery stage, the advent of robust and rapid analytical platforms/workflows has become critical. Commonly, metabolic stability data are generated using LC-MS/MS, which require both labor and instrument time to develop methods for each compound independently before analysis. Due to advances in both chromatography and mass spectrometer technology, the UFast-MS method will be widely accepted by the industry as the standard way to analyze metabolic stability. By allowing labs to forgo the traditional method development process during early drug discovery, discovery groups will be able to generate high-quality data in a much shorter time frame, increasing both throughput and productivity.

\section{Supplementary data}

To view the supplementary data that accompany this paper please visit the journal website at: www.future-science.com/ doi/full/10.4155/bio-2016-0187

\section{Acknowledgements}

We sincerely thank Ping Ling and Graciela Ternero in the Celgene IT department for their help in developing the Microsoft Excel parser to help with data analysis. We would also like to thank Waters Corporation for their technical help through- 
out this research. We would also like to thank the Celgene Medicinal Chemistry department for providing all the internal compounds used for this manuscript.

Financial \& competing interests disclosure

The authors are employees and shareholders of Celgene Corporation. The authors have no other relevant affiliations or financial involvement with any organization or entity with a financial interest in or financial conflict with the subject mat- ter or materials discussed in the manuscript apart from those disclosed.

No writing assistance was utilized in the production of this manuscript.

\section{Open access}

This work is licensed under the Attribution-NonCommercialNoDerivatives 4.0 Unported License. To view a copy of this license, visit http://creativecommons.org/licenses/by-nc-nd/4.0/

\section{Executive summary}

\section{Background}

- In vitro drug metabolism and pharmacokinetics screens, specifically metabolic stability, have been developed and have shown to be very effective in guiding candidate optimization and preventing selection of compounds with poor 'drug-like' properties.

- Metabolic stability data are universally analyzed using LC-MS techniques, predominately coupling triple quadrupole mass analyzers.

- We have developed an ultra-fast, generic analytical method for metabolic stability using high-resolution TOF-MS coupled with ballistic gradient LC and automated data processing.

Results \& conclusion

- We successfully developed a high-throughput bioanalytical workflow.

- By utilizing ballistic gradient techniques, we were able to develop a sub 1 min LC gradient chromatographic method coupled with high resolution TOF-MS for the analysis of metabolic stability samples.

- This newly developed generic method has the ability to analyze the stability of 96 compounds in quadruplicate across two species in $24 \mathrm{~h}$ and removes the need for method optimization that is required in traditionally MS/MS methods.

- Automated data processing allows for rapid integration and interpretation of all data produced and eliminated the need for manual data processing associated with traditional triple quad approaches.

\section{Conclusion}

- An advanced bioanalytical workflow for metabolic stability has been developed that demonstrates significant advantages over traditional triple quadrupole based methods, including significant increases in throughput, data processing capabilities and overall data quality.

\section{References}

Papers of special note have been highlighted as:

- of interest; $\bullet$ of considerable interest

1 O’Connor D, Mortishire-Smith R, Davies A, Dominguez M. Ultra-performance liquid chromatography coupled to time-of-flight mass spectrometry for robust, high-throughput quantitative analysis of an automated metabolic stability assay, with simultaneous determination of metabolic data. Rapid Commun. Mass Spectrom. 20(5), 851-857 (2006).

-• First demonstrated the use of ballistic LC with exact mass TOF for the analysis of metabolic stability samples. We have furthered this approach by decreasing analytical run time to sub $1 \mathrm{~min}$ as well as introducing a new data processing tool that has increased our throughput.

2 Masimirembwa CM, Thompson R, Anderson TB. In vitro high throughput screening of compounds for favorable metabolic properties in drug discovery. Comb. Chem. High Throughput Screen. 4(3), 245-263 (2001).

3 Chovan LE, Blank-Schaefer C, Dandliker PJ, Lau YY. Automatic mass spectrometry method development for drug discovery: application in metabolic stability assays. Rapid Commun. Mass Spectrom. 18(24), 3105-3112 (2004).
4 Di L, Kerns EH, Gao N et al. Automatic mass spectrometry method development for drug discovery: application in metabolic stability assays. J. Pharm. Sci. 93(6), 1537-1544 (2004).

5 Plant N. Strategies for using in vitro screens in drug metabolism. Drug Discov. Today 9(7), 328 (2004).

6 Moghaddam MF, Tang Y, O’Brien Z et al. A proposed screening paradigm for discovery of covalent inhibitor drugs. Drug. Metab. Lett. 8(1), 19-30 (2014).

7 Ackley DC, Rockish KT, Baker TR. Methods in Pharmacology and Toxicology Optimization in Drug Discovery (In vitro methods). Yan Z, Caldwell GW (Eds). Humana Press Inc., NJ, USA, 151-164 (2004).

8 National Library of Medicine. National Institutes of Health. www.ncbi.nlm.nih.gov

9 Apollonio LG, Pianca DJ, Whittall IR, Maher WA, Kyd JM. A demonstration of the use of ultra-performance liquid chromatography-mass spectrometry [UPLC/MS] in the determination of amphetamine-type substances and ketamine for forensic and toxicological analysis. J. Chromatogr. B Analyt. Technol. Biomed. Life Sci. 836(1-2), 111-115 (2006). 
10 Al-Dirbashi OY, Aboul-Enein HY, Jacob M, Al-Qahtani K, Rashed MS. UPLC-MS/MS determination of doxazosine in human plasma. Anal. Bioanal. Chem. 385(8), 1439-1443 (2006).

11 Shen JX, Wang H, Tadros S, Hayes RN. Orthogonal extraction/chromatography and UPLC, two powerful new techniques for bioanalytical quantitation of desloratadine and 3-hydroxydesloratadine at $25 \mathrm{pg} / \mathrm{ml}$. J. Pharm. Biomed. Anal. 40(3), 689-706 (2006).

12 Romanyshy L, Tiller PR, Alvaro R, Pereira A, Hop CECA. Ultra-fast gradients vs. fast isocratic chromatography in bioanalytical quantification by liquid chromatography/ tandem mass spectrometry. Rapid Commun. Mass Spectrom. 15(5), 313-319 (2001).

13 DeNardi C, Bonelli F. Moving from fast to ballistic gradient in liquid chromatography/tandem mass spectrometry pharmaceutical bioanalysis: matrix effect and chromatographic evaluation. Rapid Commun. Mass Spectrom. 20(18), 2709-2716 (2006).

14 Nassar AF, Talaat RE. Strategies for dealing with metabolite elucidation in drug discovery and development. Drug Discov. Today 9(7), 317-327 (2004).

15 Nassar AF, Kamel AM, Clarimont C. Improving the decision-making process in structural modification of drug candidates: reducing toxicity. Drug Discov. Today 9(24), 1055-1064 (2004).

16 Lave T, Dupin S, Schmitt SC, Chou RC, Jaeck D, Coassolo P. Integration of in vitro data into allometric scaling to predict hepatic metabolic clearance in man: application to ten extensively metabolized drugs. J. Pharm. Sci. 86(5), 584-590 (1997).

17 Iwatsubo T, Hirota N, Ooie T et al. Prediction of in vivo drug metabolism in the human liver from in vitro metabolism data. Pharmacol. Ther. 73(2), 147-171 (1999).

18 Xu R, Manuel M, Cramlett J, Kassel DB. A high throughput metabolic stability screening workflow with automated assessment of data quality in pharmaceutical industry. J. Chromatogr. A 1217, 1616-1625 (2010).

19 Bateman KP, Castro-Perez J, Wrona M et al. MSE with mass defect filtering for in vitro and in vivo metabolite identification. Rapid Commun. Mass Spectrom. 21(9), 1485-1496 (2007).

20 Tiller PR, Yu S, Castro-Perez J, Fillgrove KL, Baillie TA. High-throughput, accurate mass liquid chromatography/ tandem mass spectrometry on a quadrupole time-of-flight system as a 'first-line' approach for metabolite identification studies. Rapid Commun. Mass Spectrom. 22(7), 1053-1061 (2008).

21 Zhu M, Ma L, Zhang D et al. Detection and characterization of metabolites in biological matrices using mass defect filtering of liquid chromatography/high resolution mass spectrometry data. Drug Metab. Dispos. 34(10), 1722-1733 (2006).

22 Nägele E, Fandino AS. Simultaneous determination of metabolic stability and identification of buspirone metabolites using multiple column fast liquid chromatography time-offlight mass spectrometry. J. Chromatogr. A 1156, 196-200 (2007).

23 Richardson SJ, Bai A, Kulkarni AA, Moghaddam MF. Efficiency in drug discovery: liver $S 9$ fraction assay as a screen for metabolic stability. Drug Metab. Lett. 10(2), 83-90 (2016).

- Demonstrates the importance of using $S 9$ fraction metabolic stability data over microsomal stability.

24 Goshawk J, Eatough D, Wood M. Componentization following 3D-peak detection in the UNIFI scientific information system. Waters White Papers.

- Highlights how the data processing software works.

25 Leeson PD, Springthorpe B. The influence of drug-like concepts on decision-making in medicinal chemistry. Nat. Rev. 6, 881-890 (2007).

26 Vieth M, Siegel MG, Higgs RE et al. Characteristic physical properties and structural fragments of marketed oral drugs. J. Med. Chem. 47(1), 224-232 (2004).

27 Morphy R. The influence of target family and functional activity on the physicochemical properties of pre-clinical compounds. J. Med. Chem. 49(10), 2969-2978 (2006).

28 Kulkarni AA, Riggs J, Phan C et al. Proposing advancement criteria for efficient DMPK triage of new chemical entities. Future Med. Chem. 6(2), 131-139 (2014). 